\title{
Langmuir
}

pubs.acs.org/Langmuir

(C) 2010 American Chemical Society

\section{Film Thickness Dependence of Phase Separation and Dewetting Behaviors in PMMA/SAN Blend Films}

\author{
Jichun You, ${ }^{\dagger}$ Yonggui Liao, ${ }^{*}$ Yongfeng Men,${ }^{\dagger}$ Tongfei Shi, ${ }^{*}{ }^{\dagger}$ and Lijia An*, ${ }^{*}$ \\ ${ }^{\dagger}$ State Key Laboratory of Polymer Physics and Chemistry, Changchun Institute of Applied Chemistry, Chinese \\ Academy of Sciences, Changchun 130022, China, and "School of Chemistry and Chemical Engineering, \\ Huazhong University of Science and Technology, Wuhan 430074, China
}

Received March 16, 2010. Revised Manuscript Received August 4, 2010

\begin{abstract}
Film thickness dependence of complex behaviors coupled by phase separation and dewetting in blend [poly(methyl methacrylate) (PMMA) and poly(styrene-ran-acrylonitrile) (SAN)] films on silicon oxide substrate at $175{ }^{\circ} \mathrm{C}$ was investigated by grazing incidence ultrasmall-angle X-ray scattering (GIUSAX) and in situ atomic force microscopy (AFM). It was found that the dewetting pathway was under the control of the parameter $U_{\mathrm{q} 0} / E$, which described the initial amplitude of the surface undulation and original thickness of film, respectively. Furthermore, our results showed that interplay between phase separation and dewetting depended crucially on film thickness. Three mechanisms including dewetting-phase separation/wetting, dewetting/wetting-phase separation, and phase separation/wettingpseudodewetting were discussed in detail. In conclusion, it is relative rates of phase separation and dewetting that dominate the interplay between them.
\end{abstract}

\section{Introduction}

Polymer blend films have attracted intense interest because of scientific interests as well as industry applications in recent decades. ${ }^{1-4}$ These applications and properties are mainly controlled by basic phase behaviors. On one hand, many researchers have focused on phase separation, since they were blends. Structures and dynamics of phase separation have been investigated systematically. ${ }^{5-7}$ It is found that they are dominated by many factors such as molecule weight, temperature, and substrate. In particular, film thickness dependence has received too much attention. In some cases, however, significant discrepancies have been found. For instance, Binder and his co-workers ${ }^{8}$ proved in theory that miscibility and critical temperature increased with decreasing films thickness, which resulted in slow kinetics of phase separation. However, results of Tanaka et al. ${ }^{9}$ showed that lower critical solution temperature (LCST) of polystyrene-poly(viny1 methyl ether) (PSPVME) blend films changed from more than $450 \mathrm{~K}$ to room temperature when film thickness decreased from $1500 \mathrm{~nm}$ to $25 \mathrm{~nm}$. Thus, it is necessary to clarify this problem in our system not only for the purposes of phase separation itself, but also for further investigation of complex behaviors, e.g., interplay between phase separation and dewetting.

On the other hand, dewetting of polymer film has been studied for several decades due to the advantages of the polymer as a

\footnotetext{
*To whom correspondence should be addressed. E-mail: tfshi@ ciac.jl.cn or ljan@ciac.jl.cn. Tel: +86-431-85262137. Fax: +86-431-85262969.

(1) Geoghegan, M.; Krausch, G. Prog. Polym. Sci. 2003, 28, 261.

(2) Wang, L.; Hong, S.; Hu, H. Q.; Zhao, J.; Han, C. C. Langmuir 2007, 23, 2304

(3) Tabatabaei, S. H.; Carreau, P. J.; Ajji, A. J. Membr. Sci. 2008, 325, 772

(4) Chen, L. M.; Hong, Z. R.; Li, G.; Yang, Y. Adv. Mater. 2009, 21, 1434.

(5) Budkowski, A. Adv. Polym. Sci. 1999, $148,1$.

(6) Takeno, H.; Hashimoto, T. J. Chem. Phys. 1998, 108, 1225

(7) Nisato, G.; Ermi, B. D.; Douglas, J. F.; Karim, A. Macromolecules 1999, 32 , 2356.

(8) Binder, K. Adv. Polym. Sci. 1999, 138, 1 .

(9) Tanaka, K.; Yoon, J. S.; Takahara, A.; Kajiyama, T. Macromolecules 1995, 28,934 .

(10) Liao, Y. G.; Su, Z. H.; Sun, Z. Y.; Shi, T. F.; An, L. J. Macromol. Rapid Commun. 2006, 27, 351 .

(11) Reiter, G. Phys. Rev. Lett. 1992, 68, 75
}

model. ${ }^{10,11}$ There are several dewetting mechanisms including spinodal dewetting, nucleation and growth, autophobicity, and composition (density) fluctuation. ${ }^{12}$ Many factors such as molecule weight, substrate, and external conditions affect structures and dynamics of dewetting. Obviously, film thickness plays an important role in them. Sharma suggested that there was a critical thickness in single-component films. Furthermore, pathway and morphologies of dewetting were different, which was ascribed to the form of intermolecular potential in an extended neighborhood of the initial thickness. ${ }^{13,14}$ On the basis of the composition fluctuation introduced by Wensink, ${ }^{12}$ the parameter of $U_{\mathrm{q} 0} / E$, which described the initial amplitude of the surface undulation and original thickness of film, respectively, was employed to distinguish the pathway and morphologies of spontaneous dewetting in PMMA/SAN blend films in our previous work. ${ }^{15}$ It was found that, for film with higher magnitude of $U_{\mathrm{q} 0} / E$, it dewetted by means of "fragment of bicontinuous structure", while for film with lower magnitude of $U_{\mathrm{q} 0} / E$, it underwent the process of "expansion of holes". Transition between them has been carried out by varying the composition in the blend (with different $U_{\mathrm{q} 0}$ ). According to this law, it is expected that transition from one mechanism to the other will take place when film thickness is varied (with different $E$ ).

Much effort has been devoted to investigating the interplay between phase separation and dewetting. For instance, Kanemoto et al. ${ }^{16}$ recently reported how fluorescence spectroscopy and AFM can be used to understand the phase separation of nanoparticles embedded in polybutadiene thin films as a function of dewetting and film rupture. This is a very smart way to clarify the complex phase behaviors of "phase separation of nanoparticles and polymer" and dewetting of thin film. However, in the case of

(12) Wensink, K. D. F.; Jérôme, B. Langmuir 2002, 18, 413.

(13) Sharma, A.; Khanna, R. Phys. Rev. Lett. 1998, 81, 3463.

(14) Sharma, A.; Khanna, R. J. Chem. Phys. 1999, 110, 4929.

(15) You, J. C.; Hu, S. S.; Liao, Y. G.; Song, K. X.; Men, Y. F.; Shi, T. F.; An, L. J. Polymer 2009, 50, 4745.

(16) Kanemoto, R.; Anas, A.; Matsumoto, Y.; Ueji, R.; Itoh, T.; Baba, Y.; Nakanishi, S.; Ishikawa, M.; Biju, V. J. Phys. Chem. C 2008, 112, 8184. 
polymer blend films, there are many incongruous mechanisms including phase separation/bilayer-dewetting ("/"means taking place at the same time and "-" means followed by another process, sic passim), dewetting-phase separation/wetting., ${ }^{9,10,17-22}$ It seems that they cannot coexist in one system. In this work, we try to unify kinds of interplay mechanisms by varying thickness, since it plays an important role both in phase separation and in dewetting. Our results revealed that relative rates of these two phase behaviors were the key factor of interplay between them. Different mechanisms discussed above can be regarded as embodiment of relative rate dependence.

\section{Experimental Section}

Materials. The polymer system is a blend of poly(methyl methacrylate) (PMMA) $\left(M_{\mathrm{w}}=387 \mathrm{~kg} / \mathrm{mol}, \mathrm{PDI}=3.72\right)$ and ploy(styrene-ran-acrylonitrile) $(\mathrm{SAN})\left(M_{\mathrm{w}}=149 \mathrm{~kg} / \mathrm{mol}, \mathrm{PDI}=\right.$ $2.66,30 \mathrm{wt} \%$ of AN) purchased from Across and Aldrich, respectively.

Sample Preparation. Silicon wafers were cleaned in a bath of $100 \mathrm{~mL}$ of $80 \% \mathrm{H}_{2} \mathrm{SO}_{4}, 35 \mathrm{~mL}$ of $30 \% \mathrm{H}_{2} \mathrm{O}_{2}$, and $15 \mathrm{~mL}$ of deionized water at $80^{\circ} \mathrm{C}$ for $60 \mathrm{~min}$ and rinsed in deionized water, then blown dry with compressed nitrogen. Thin blend films were prepared by spin-coating 1,2-dichloroethane solution (with different concentration) of PMMA/SAN (50:50 wt \%) onto the cleaned silicon wafer. After preparation, films were kept under vacuum for $12 \mathrm{~h}$. Thicknesses measured by a D8 Discover X-ray reflectometer (Bruker, Germany) were $8.9 \mathrm{~nm}$ (labeled as S89), $39.8 \mathrm{~nm}$ (labeled as S398), and $50.4 \mathrm{~nm}$ (labeled as S504), respectively.

AFM and GIUSAX Measurements. The in situ atomic force microscopy (AFM) topography images were obtained using an SPA-300 (Seiko Instruments Inc., Japan) at $175^{\circ} \mathrm{C}$ with a temperature-control stage driven in tapping mode. A silicon tip (Olympus, Japan) with a spring constant of $42 \mathrm{~N} / \mathrm{m}$ was used. Synchrotron grazing incidence ultrasmall-angle X-ray scattering (GIUSAX) measurements were performed at the BW4 beamline at HASYLAB (DESY, Germany), using a monochromatic X-ray beam with energy of $8.979 \mathrm{keV}$. The sample to detector distance was $L=13037 \mathrm{~mm}$. X-ray scattering intensity patterns were acquired by a two-dimensional detector array $(2048 \times 2048$ pixels $)$. Prior to measurement of GIUSAX, samples were annealed at $175^{\circ} \mathrm{C}$ (higher than bulk phase separation temperature, $160{ }^{\circ} \mathrm{C}^{23}$ ) for different times in the oven, respectively.

\section{Result and Discussion}

In our previous work, ${ }^{22}$ we only found that film thickness can strongly influence phase separation and dewetting. However, there is no suitable method to distinguish phase separation from dewetting when both of them take place, which hinders further understanding of interplay between them. In addition, in another previous work, ${ }^{15}$ we successfully established the combination method of AFM and GIUSAX, which can identify both behaviors and do some further investigation on dewetting induced by composition fluctuation. Therefore, the above results make it possible for us to investigate the complex interplay between phase separation and dewetting in this work.

(17) Müller-Buschbaum, P.; O’Neill, S. A.; Affrossman, S.; Stamm, M. Macromolecules 1998, 31, 5003 .

(18) Yerushalmi-Rozen, R.; Kerle, T.; Klein, J. Science 1999, 285, 1254

(19) Oron, M.; Kerle, T.; Yerushalmi-Rozen, R.; Klein, J. Phys. Rev. Lett. 2004, $92,236104$.

(20) Clarke, N. Macromolecules 2005, 38, 6775.

(21) Müller-Buschbaum, P.; Bauer, E.; Wunnicke, O.; Stamm, M. J. Phys: Condens. Matter. 2005, 17, s363.

(22) Liao, Y. G.; You, J. C.; Shi, T. F.; An, L. J.; Dutta, P. K. Langmuir 2002, 23, 11107.

(23) Wang, H.; Composto, R. J. Interface Sci. 2003, 11, 237.
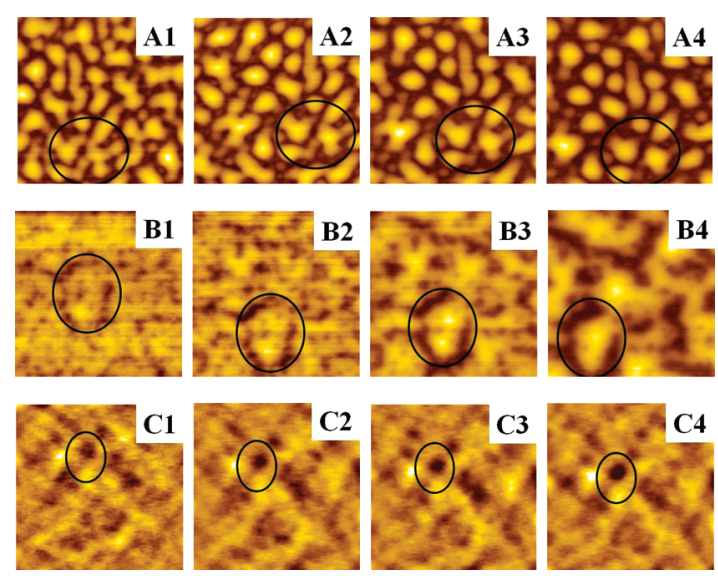

Figure 1. Snapshots of topography images for PMMA/SAN $(50: 50 \mathrm{wt} \%$ ) thin films (A for S89, B for S398, and C for S504) at $175^{\circ} \mathrm{C}$ for A: $145,219,257,338 \mathrm{~min}$. B: $24,63,180,358 \mathrm{~min}$. C: 16 , $40,54,92 \mathrm{~min}$. All dimensions are $2 \times 2 \mu \mathrm{m}^{2}$.

Thickness Dependence of Dewetting Pathway. First of all, it is necessary to introduce composition fluctuation (predicted by Wensink et al. ${ }^{12}$ ) in brief, since it is the theoretical support of dewetting in our work. Spin-coated PMMA/SAN film is homogeneous, since the solvent evaporates too quickly for polymer chains to reconstruct. When the film is heated above its glass transition temperature, PMMA diffuses to the substrate so as to create the composition gradient due to the favorable interaction with the substrate. ${ }^{23}$ Since this diffusion cannot take place precisely in the same way over the whole film, this is likely to create fluctuation in the mixture composition along the surface. When the amplitude of fluctuation is large enough, the free surface will be destabilized eventually. This dewetting mechanism has been predicted and validated by theory ${ }^{12}$ and experiment, ${ }^{15}$ respectively.

Row A in Figure 1 shows the evolution of S89 during dewetting at $175^{\circ} \mathrm{C}$. In Figure $1 \mathrm{~A} 1$, we can find some bicontinuous structures (highlighted by ellipse) which are similar to the bicontinuous phase structures of phase separation in polymer blend films. ${ }^{24}$ With further annealing, these structures with long hills and gullies grow and develop in the direction of surface and thickness. From then on, more and more parts of the structure (top-left part in Figure 1A2, top-right and bottom-left parts in ellipse of Figure 1A3) decay from the main one, resulting in the formation of droplets. Then, these droplets become rounder and rounder due to surface tension. This process is the reoccurrence of the dewetting pathway of SAN50 with nearly equal thickness annealed at $145^{\circ} \mathrm{C}$ in our previous work. ${ }^{15}$ It is worth noting that the scenario highlighted in Figure $1 \mathrm{~A}$ is the epitome of transition from bicontinuous structure to droplet in the whole scanned area. This is the so-called "fragmentation of bicontinuous structures". Results of S504 (Figure 1C) show a completely different pathway. It is easy to find that there are some holes surrounded by uneven rims on the surface when the film is heated at $175^{\circ} \mathrm{C}$ for $145 \mathrm{~min}$ (Figure 1C1). They grow in height and diameter while the number of holes does not increase remarkably with further annealing. A typical example is highlighted by the ellipse in Figure 1C. Seemingly, this scenario is similar to the dewetting pathway of SAN90 annealed at $145^{\circ} \mathrm{C}$ in our previous work. ${ }^{15}$ It is obvious that results of S398 are the mixture of the above two mechanisms. Both holes and bicontinuous structures appear in Figure 1B1. However, the former develops so rapidly that the latter is invisible in the following

(24) Affrossman, S.; Henn, G.; O’Neill, S. A.; Pethrich, R. A.; Stamm, M. Macromolecules 1996, 29, 5010. 


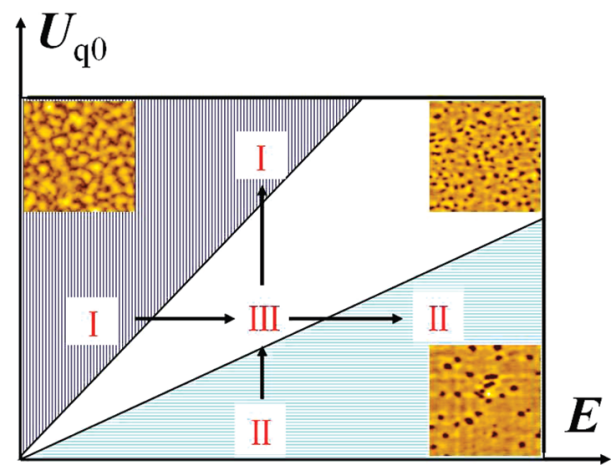

Figure 2. Schematic representations of morphologies and pathway during dewetting corresponding to different value of $U_{\mathrm{q} 0}$ and $E$.

images (Figure 1B2). During above evolution, many new holes occur, leading to the significant increase of hole number per unit area (Figure 1B2 and B3). Then, the holes have no choice but to merge with each other. The merger of neighboring holes results in the occurrence of "valley" (Figure 1B4). It is crossover or growth in certain direction of these valleys that leads to the formation of island structures. Obviously, the area highlighted by ellipse in Figure 1B1-4 is a copy of the dewetting pathway of SAN70 in our previous work. ${ }^{15}$ It is named the "transitional pathway". From the above discussion, it is indicated that dewetting (or pseudodewetting) pathways of samples with thicknesses of $50.4 \mathrm{~nm}$, $39.8 \mathrm{~nm}$, and $8.9 \mathrm{~nm}$ are similar to the results of SAN90, SAN70, and SAN50 in our previous work, respectively. In that work, two kinds of dewetting pathway and their transition were carried out by varying the composition of blend. It was found that the ratio of $U_{\mathrm{q} 0} / E$ played an important role in the pathway of dewetting. We can conclude those results in Figure 2 (including change in $E$ and $\left.U_{\mathrm{q} 0}\right)$. There are three regions in this figure. In region I, a higher ratio of $U_{\mathrm{q} 0} / E$ (higher $U_{\mathrm{q} 0}$, lower $E$ ) is the reason for "fragmentation of bicontinuous structures". In region II, the ratio of $U_{\mathrm{q} 0} / E$ is lower (lower $U_{\mathrm{q} 0}$, higher $E$ ), leading to the occurrence of "expansion of holes". Of course, transitional morphologies and pathway of dewetting can be obtained in region III. Change of either $U_{\mathrm{q} 0}$ or $E$ can result in the variation of this ratio. In our previous work, the transition (in vertical direction in Figure 2) between two kinds of dewetting pathway was carried out using a different original intensity in composition fluctuation resulting from a different composition in the blend. In this work, the parameter $U_{\mathrm{q} 0} / E$ is validated by a different thickness in blend films, which is a transition in the horizontal direction in Figure 2 (with approximate $U_{\mathrm{q} 0}{ }^{25}$ but different $E$ ). Results indicate that it works as well as that in single-component films. ${ }^{13,14,26}$

Figure 3 shows the annealing time dependence of root-meansquare roughness (RMSr) obtained from topography images (by commercial software equipped with AFM) during dewetting of three samples. It is worth noting that RMSr depends on the scanned area. In other words, values of RMSr are different in different regions. Therefore, it is hard to show all measured RMSr data here. However, in our experiment, the scopes of RMSr development are approximate. RMSr of three samples increases when they are annealed at $175^{\circ} \mathrm{C}$; however, there is great difference in the increase of them. In our previous publication, ${ }^{22}$ they were regarded as irrelevant power exponents including $0.18,0.45$, and 0.37 . In this paper, we will discuss them in a new way: in the result

(25) Sferrazza, M.; Xiao, C.; Jones, A. L. Phys. Rev. Lett. 1997, 78, 3693.

(26) Xie, R.; Karim, A.; Douglas, J. F.; Han, C. C.; Weiss, R. A. Phys. Rev. Lett. 1998, $81,1251$.

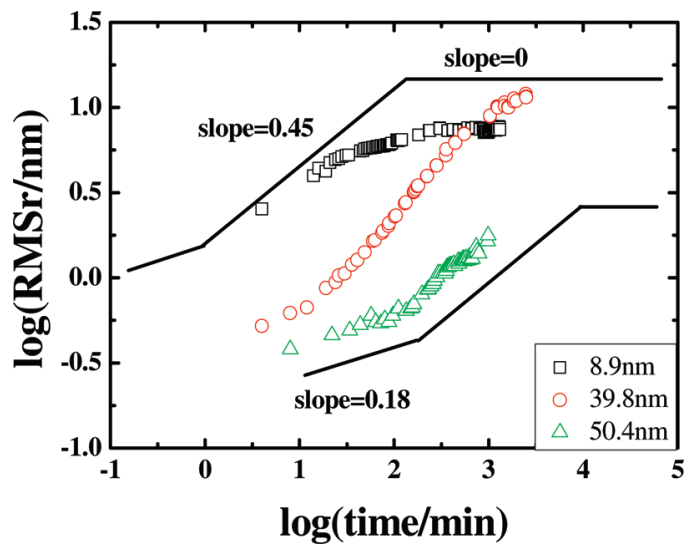

Figure 3. Annealing time dependence of RMSr (root-meansquare of roughness) in topography images during dewetting in samples of S89, S398, and S504.

of S504, the increase of RMSr follows the power law of $t^{0.18}$ and $t^{0.45}$ in the early and later stage, respectively; the power exponent tends to a very low magnitude from $\sim 200 \mathrm{~min}$ and can be treated as zero in S89; the result of S398 is similar to that in the thicker one. However, we can find a shorter early stage and the rudiment of the later stage in which the exponent tends to zero. We can conclude the above discussion as follows: there are three stages of RMSr evolution during dewetting (lines shown in Figure 3), similar to the early, intermediate, and late stages (exponents of RMSr increase in these stages are $0.18,0.45$, and 0 , respectively) in spinodal dewetting ${ }^{15,25,27}$ and spinodal decomposition. ${ }^{5,28}$ These similarities come from the relativity of them. ${ }^{29-32}$ However, exponents in our experiments do not fit the exponential growth theory predicted by Brochard and Dalliant. ${ }^{33,34}$ One possible reason for this difference is the movement of PMMA to the substrate due to the strong interaction between them, which has been discussed in our previous publication. ${ }^{35}$ We can find the first and second stages in the result of S504, since slow kinetics has been obtained in the above discussion. During the dewetting of the transitional sample (S398), the end of the first stage, the whole second stage, and the rudiment of the third stage are observed. However, only a very short end of the second stage and the third stage appear in the thinner sample (S89) due to the rapid dewetting resulting from thinner film and high loss of conformational entropy of polymer chains. ${ }^{10}$ We can go back to Figure 1 and compare Figure 1A4 and B4. Droplets in A4 are clear at $92 \mathrm{~min}$, while rudiments of them begin to appear even in $358 \mathrm{~min}$ in B4, suggesting that dewetting takew place more easily in films with lower thickness. In other words, it takes more (less) time to complete dewetting in thicker (thinner) films. Relative to our previous publication, ${ }^{22}$ this discussion is completely new and provides a different way to understand the dewetting process caused by composition fluctuation.

Interplay between Phase Separation and Dewetting. To detect phase-separated structures in these films, it is necessary to perform the measurement of GIUSAX, a powerful method which

(27) Brochard, W. F.; Martin, P.; Redon, C. Langmuir 1993, 9, 3682

(28) Polymer Blend, Paul, D. R.; Newman, S., Eds.; Academic Press: New York, 1978

(29) Sharma, A.; Mittal, J. Phys. Rev. Lett. 2002, 89, 186101

(30) Kaya, H.; Jérôme, B. Eur. Phys. J. E. 2003, 12, 383

(31) Green, P. F.; Ganesan, V. Eur. Phys. J. E. 2003, 12, 449.

(32) Sharma, A.; Mittal, J; Verma, R. Langmuir 2002, 18, 10213

(33) Brochard, F.; Daillant, J. Can. J. Phys. 1990, 68, 1084.

(34) Milchev, A.; Binder, K. J. Chem. Phys. 1997, 106, 1978

(35) You, J. C.; Shi, T. F.; Liao, Y. G.; Li, X. L.; Su, Z. H.; An, L. J. Polymer 2008, 49, 4456. 

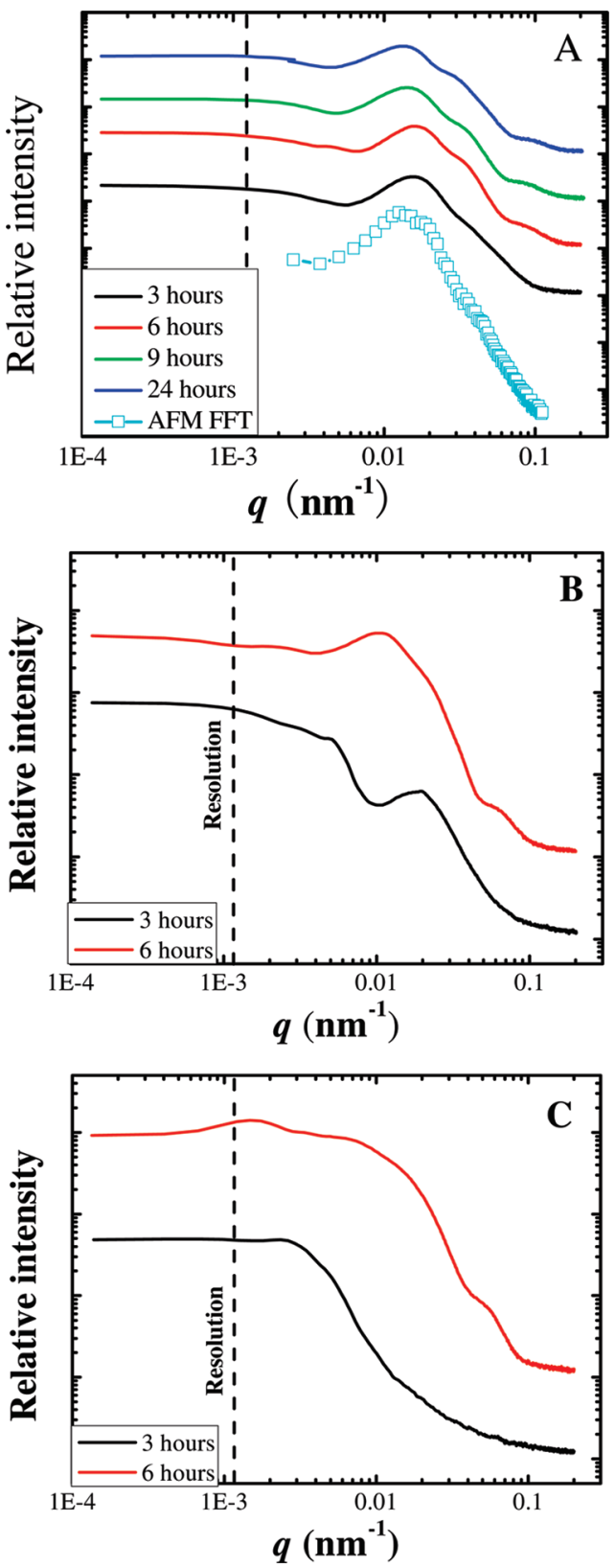

Figure 4. Common double logarithmic presentation in reciprocal space of GIUSAX of samples with thickness of S89 (A), S398 (B), and S504 (C). Lines are results of GIUSAX, and light blue triangle curve in $\mathrm{A}$ is the fast Fourier transition (FFT) from AFM images $\left(5 \times 5 \mu \mathrm{m}^{2}\right)$ annealed at $175{ }^{\circ} \mathrm{C}$ for $800 \mathrm{~min}$. Dashed line is the resolution of GIUSAX.

has been employed by many researchers in the investigation of (micro)phase separation. ${ }^{15,36-39}$ In Figure 4A (S89), lines are results of GIUSAX; the light blue triangle curve is obtained by fast Fourier transition (FFT) from AFM images $\left(5 \times 5 \mu \mathrm{m}^{2}\right)$ annealed at $175^{\circ} \mathrm{C}$ for $800 \mathrm{~min}$ (AFM data not shown here). FFT curve shows the correlation of droplets resulting from dewetting. From the comparison of the black and light blue triangle curves, it is clear that there are two peaks with lower magnitude of $q$,

(36) Müller-Buschbaum, P.; Gutmann, J. S.; Stamm, M.; Cubitt, R.; Cunis, S.; von Krosigk, G.; Gehrke, R.; Petry, W. Physica B 2000, 283, 53.

(37) Müller-Buschbaum, P.; Cubitt, R.; Petry, W. Langmuir 2003, 19, 7778

(38) Lee, B.; Yoon, J.; Oh, W.; Hwang, Y.; Heo, K.; Jin, K. S.; Kim, J.; Kim, K. W.; Ree, M. Macromolecules 2005, 38, 3395.

(39) Sun, Z.; Wolkenhauer, M.; Bumbu, G. G.; Kim, D. H.; Gutmann, J. S. Physica B 2005, 357, 141. corresponding to two kinds of ordered structures. They are the representation of correlation among droplets with different distances, which is the reason for similar peaks in GIUSAX and FFT curves. The third one $\left(q=0.093 \mathrm{~nm}^{-1}\right)$ comes into being with further annealing to $6 \mathrm{~h}$ (shown in the red line in Figure 4A). It is not caused by morphologies, since it does not exist in the FFT curve. As shown in many reports, ${ }^{15,36}$ it is the result of correlation among phase-separated structures (PMMA-rich and SAN-rich phases) in the droplets. Then, it is confirmed that dewetted structures are detected at $3 \mathrm{~h}$, while phase-separated structures can be observed from GIUSAX results until $6 \mathrm{~h}$. In other word, dewetting takes place earlier than phase separation in this sample (S89). There is no obvious change in the peak or its position (green and dark blue lines in Figure 4A), which means that dewetted and phase-separated structures are stable with further annealing. In the same way, these three peaks with close magnitude of $q$ can be found in Figure 4B. Similarly, we can find only dewetted structures without phase-separated structures when the samples are annealed for $3 \mathrm{~h}$. The later structures come into being between 3 to $6 \mathrm{~h}$. In Figure 4C, neither dewetted nor phase-separated structure is visible with $3 \mathrm{~h}$ annealing. We can find both of them in the red line (annealed for $6 \mathrm{~h}$ ). However, the intensity of dewetting peaks becomes so weak that it is hard to detect in Figure 4C. Conversely, the peak of phase separation is strengthened relative to the former one. Two points can be obtained from the variation of intensity: one, dewetting becomes slower due to the increase of film thickness, which is in good agreement with the evolution of RMSr shown in Figure 3; the other, phase separation is the ascendant behavior in $6 \mathrm{~h}$. It can be expected that phase-separated structures will appear earlier than dewetted ones in films with higher thickness. There is no obvious variation in the appearance time of phase separation peaks in Figure 4A,B,C, indicating that the rate of phase separation in our blend films is not sensitive to film thickness on our experiment time scale, which is in good agreement with results from Chung et al. ${ }^{40}$ in a similar blend of PMMA/SAN. However, it is completely different from the result of PS/PVME blend films from Tanaka et al. ${ }^{9} \mathrm{~A}$ possible reason is the difference of blend systems or film confinement. ${ }^{40}$

As a matter of fact, there are both influence and competition with each other between phase separation and dewetting in polymer blend films. ${ }^{41}$ On one hand, variation of critical temperature and kinetics are the embodiment of the influence of dewetting on phase separation. ${ }^{9,15}$ On the other hand, one of the behaviors (phase separation or dewetting) will take place earlier and faster than the other due to the competition between them. According to above discussion in our system (thickness dependence of kinetics of phase separation and dewetting), it is clear that dewetting becomes slower while phase separation is insensitive to increasing film thickness. Film thickness dependence on the interplay between phase separation and dewetting can be shown as Figure 5. The dash and dash-dot lines represent the relative rates of dewetting and phase separation, respectively. Then, we can get three different regions due to the crossover of two lines. In region $I$, the occurrence of dewetted structures is earlier than phase-separated structures, since the relative rate of dewetting is higher, resulting in the mechanism of "dewetting-phase separation/wetting" reported in our previous work. 9 In region III, dewetting is slower because of the significant increase of film thickness, while the rate of phase separation does not vary remarkably. As a result of this, phase-separated structures can be observed earlier than dewetted structures. Or in the more

(40) Chung, H.; Composto, R. J. Phys. Rev. Lett. 2004, 92, 185704

(41) Ogawa, H.; Kanaya, T.; Nishida, K.; Matsuba, G. Polymer 2008, 49, 254. 


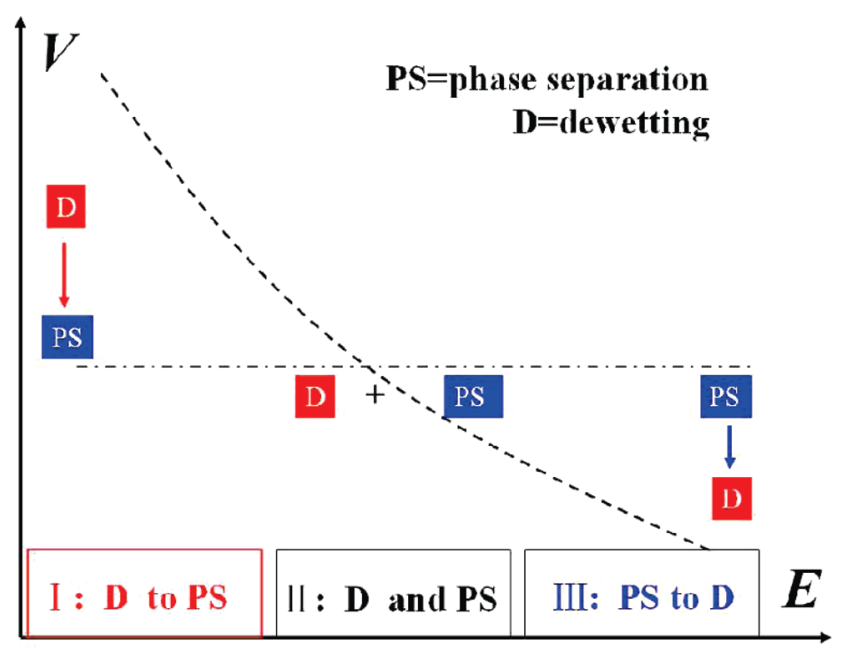

Figure 5. Schematic representations of different mechanisms during complex phase behaviors including phase separation and dewetting due to the variation of relative ratio between them.

extreme situation, dewetting will not take place at all. This is the reason for the appearance of the "phase separation/bilayerdewetting" mechanism reported and cited in the literature., In our system of PMMA/SAN, however, not phase separation/ bilayer-dewetting, but phase separation/wetting-pseudodewetting is the suitable expression, since there is no bilayer structure. There are three reasons for this: first, PMMA/SAN is a weak immiscible system; second, sizes of phase-separated and dewetted structures are not on a comparable scale (there is an especial peak of phase separation in Figure 4.); finally, wetting of PMMA is unavoidable and preferential, since it is the foundation of composition fluctuation. In region II, there will be complex phase behaviors, since phase separation and dewetting take place at approximate rates. They will dominate the final structure simultaneously. Then, what is the interplay mechanism among phase separation of PMMA and SAN, dewetting of the whole film, and preferential wetting of the PMMA layer on the substrate in this film thickness region? It is clear that, in Figure 3, RMSr of S398 $(\sim 10 \mathrm{~nm})$ has a much lower magnitude compare to film thickness (39.8 nm). Dewetting of S398 takes place feebly (compare to S89) and slowly (although it is still faster than phase separation) coupled by wetting of the PMMA layer on the silicon oxide substrate. We can describe our current result as "dewetting/wettingphase separation" (dewetting means the whole film, while wetting means PMMA layer to the substrate). It is completely different from the case of S89, in which the whole film dewets rapidly and completely due to the high loss of conformational entropy when it is heated to $175^{\circ} \mathrm{C}$.
In a word, the interplay between phase separation and dewetting lies in the film thickness due to the thickness dependence on the relative rate of dewetting and phase separation. This is in good agreement with the result from Ogawa et al., ${ }^{41}$ in which PS/ PVME blend films with thicknesses from $65 \mu \mathrm{m}$ (thick film) to $42 \mathrm{~nm}$ (thin film $\sim 2.5 R_{\mathrm{g}}$ ) were investigated by AFM and light scattering. However, in our system, the interaction between PMMA and substrate is stronger than that between SAN and the substrate, which makes our system more complicated, especially for our film thickness from $50.4 \mathrm{~nm}$ (thin film $\sim 5 R_{\mathrm{g}}$ ) to $8.9 \mathrm{~nm}$ (ultrathin film $\sim R_{\mathrm{g}}$ ). Namely, there will be wetting of PMMA on the substrate of silicon if samples are heated above the glass transition temperature of the two polymers, which is not only the foundation stone of dewetting induced by composition fluctuation, but also the reason for the occurrence of many complex mechanisms, e.g., "dewetting-phase separation/wetting". In other words, wetting of PMMA enriches the interplay behavior in our system. Furthermore, our results indicate that many mechanisms which look incongruous are the embodiment of the thickness dependence. They can coexist in one system and be changed from one to another by varying some factors such as composition, film thickness, miscibility of blend, and interaction with the substrate. Finally, we can draw the conclusion that it is the relative rate of phase separation and dewetting in polymer blend films that dominates the complicated interplay between them.

\section{Conclusion}

In this work, the parameter $U_{\mathrm{q} 0} / E$, introduced in our previous work, is validated by PMMA/SAN blend films with thickness from 1 to $5.5 R_{\mathrm{g}}$ (radius of gyration). It works as well as singlecomponent films with different thicknesses and blend films with different compositions. ${ }^{13-15,26}$ Furthermore, the interplay between phase separation and dewetting is investigated by PMMA/SAN blend films with different thicknesses. It is found that relative rates of them play an important role. Three regions in different thickness ranges and reasons for various mechanisms including "dewetting-phase separation/wetting", "dewetting/wetting-phase separation", and "phase separation/wetting-pseudodewetting" were discussed in detail. Our results are significant for controlling the dewetting pathway and further investigation of complex phase behaviors.

Acknowledgment. This work is supported by the National Natural Science Foundation of China (50973110, 20923003) Programs and the Fund for Creative Research Groups (50921062), and subsidized by the Special Funds for National Basic Research Program of China (2010CB631100). T.F. Shi also thanks HASYLAB project II-20052011. We thank Mr. Zhiyong Jiang, Jianqi Zhang, Ms. Shanshan Hu, Dr. A. Timmann, and Dr. S. V. Roth for assistance of GIUSAX at HASYLAB. 